\title{
JD6 -Time and Astronomy Preface and Posters
}

\author{
Pascale Defraigne
}

Royal Observatory Belgium, BE 1180 Brussels, Belgium

Responsibility for the definition of time scales left the astronomical community some 40 years ago when, in 1967, the second became defined by an atomic transition in the International System of units SI and when International Atomic Time (TAI) was defined as the primary international time scale in 1971. Atomic time is now $10^{7}$ times more stable than the universal time UT defined by the observed Earth rotation rate and some $10^{4}$ times more stable than the planetary orbital motions that were used to define time until 1967.

But time still interacts with astronomy in many ways: as the independent variable for the description of all dynamical systems, its stability allows one to study these systems and their perturbations. Time is therefore of major importance for astronomers, with time scales based on the SI second for practical applications and coordinate time scales for theoretical developments. Precise timing of the rotational and orbital periods of pulsars has the potential to contribute to the long-term stability of TAI, thereby returning some aspects of time keeping to astronomy. Furthermore, since observational techniques rely on the measurement of the time of propagation of electromagnetic signals, astronomy provides an important testing ground for relativity.

Those were the starting ideas of the Joint Discussion "Time and Astronomy" which gathered more than sixty participants during the IAU General Assembly 2009, in Rio de Janeiro. These proceedings provide a summary of all the subjects discussed during the meeting.

Supporting Commission: 31: Time

\section{Co-supporting Commissions:}

4: Ephemerides, 7: Celestial Mechanics and Dynamical Astronomy, 8: Astrometry, 19: Rotation of the Earth, 52 : Relativity in Fundamental Astronomy, 40 : Radio Astronomy.

\section{Members of the Scientific Organizing Committee:}

Pascale Defraigne (Belgium, chair), Aleksander Brzezinski (Poland, co-chair), Gambis Daniel (France), Sergei Klioner (Germany), Yury Ilyasov (Russia), Michael Kramer (UK), Richard Manchester (Australia), Demetrios Matsakis (US), Rendong Nan (China), Gerard Petit (France).

\section{List of Posters:}

Inspection for Secular Variation of Earth's Rotation Inferred from Chinese Ancient Astronomical Records,

Y. $L i$

The difference of the Earth dynamical flattening $(\mathrm{H})$ between precession observation and earth model can be reduced by $\frac{2}{3}$

C. L. Huang, Y. Liu 
Post-Newtonian Reference Frames for Advanced Theory of the Lunar Motion and a New Generation of Lunar Laser Ranging

S. Kopeikin, Y. Xie

Status and future of the cooperative San Juan SLR Station

R. Podest, L. Weidong, E. Actis, et al.

High-resolution Earth rotation parameters from the continuous VLBI campaign CONT08 M. Schindelegger, J. Boehm, S. Englich, H. Schuh

Russian astronomical yearbooks in editions and program systems

N. Glebova, G. Kosmodamianskiy, M. Lukashova, I. Netsvetaev, G. Netsvetaeva, E. Parijskaja, M. Sveshnikov, V. Skripnichenko

Design and Analysis of Nanosecond Time Synchronization System of Each Station of

Each Chain of Chinese LORAN-C H. J. Ma, Y. H. Hu, J. F. Wu, Z. M. He

Two-Way Satellite Time and Frequency Transfer Experiment via IGSO Satellite X. Yang, F. Wu, Z. Li

Recent activities at NICT Space-Time Standards Group

M. Hosokawa, K. Imamura, T. Iwama, S. Hama, J. Amagai, Y. Hanado, R. Ichikawa, Y. Koyama

Research on the technology of Common-view based on the Chinese Area Positioning System

J. F. Wu, Y. H. Hu, X. Lu, H. J. Ma, L. Hou, Z. M. He, J. Wang

Aircraft High Dynamic Two-Way Time Synchronization Technique research

H. J. Ma, Y. H. Hu, J. F. Wu, J. G. Wang

Studies on Algorithm of Autonomous Time Synchronization for Constellations

X. Lu, F. Huang, H. Wu, Y. Bian

Heterodyne Digital Method in Precision Frequency Measurement

X. $\mathrm{Li}$

The precise timing of millisecond pulsars as a possible method of the study of globular clusters and Galaxy structures

T. Larchenkova

Pulsar survey with the telescope FAST

R. Nan

Pulsar Timing package TIMAPR upgrade

O. Doroshenko

Pulsar timing as a tool for gravitational waves detection M. Sazhin

A New Algorithm for Detecting Gravitational Waves Using Pulsars

$R$. Shannon

Planetary ephemerides and pulsar timing

A. Romanoff

Pulsar Time Scale study by ensembles of simulated models

N. Korotkova, Y. Ilyasov, M. Pshirkov 